\title{
Analysis and Evaluation of the Stability of a Biologically Inspired, Leg Loss Tolerant Gait for Six- and Eight-Legged Walking Robots
}

\author{
Martin Görner and Gerd Hirzinger
}

\begin{abstract}
This article analyzes and evaluates the stability of the biologically inspired gait of the DLR Crawler, a walking hexapod robot, with respect to leg loss. Using a kinematic simulation, ranges of velocity commands that result in stable gait coordination are determined for both cases, the undamaged robot and the robot experiencing the loss of a single leg. The results give insight how to adjust the motion commands after the loss of a leg. Further, a simplified dynamic simulation is used to analyze the effect of leg loss on the walking stability. Heuristic measures like curvature and length of the traveled path, roll and pitch angles are employed to evaluate the walking stability and performance. Some methods like shifting the COG or stiffening the variably compliant joints are proposed and discussed with respect to their ability to improve the walking performance in case of leg loss. In the end, the presented concepts are extended and for the first time applied to a simulated eight-legged robot.
\end{abstract}

\section{INTRODUCTION}

For several decades now research is conducted on sixand eight-legged walking machines. This research is mainly motivated by two different goals. The first is the development of tools, which help to understand and to verify findings in biology and thus give insight in the underlying principles of locomotion. The second is to build high-performance exploration robots that show the same powerful capabilities as walking insects. Of special interest in this case are the ability of insects to negotiate rough and unstructured terrain using only distinct footholds as well as their adaptability to damage or even the loss of a leg.

Currently, different approaches exist for gait coordination of multi-legged robots. Some favor central pattern generators in combination with reflexes [1], [11], while others rely on decentralized, reactive algorithms that sense and react to the environment [10]. Common to all theses approaches is the need to be robust and to react to unforseen disturbances, thus requiring a somehow flexible coordination of the legs. A very appealing approach of a decentralized, reactive controller is the Walknet [8], [10], which incorporates findings from experiments with stick insects [2]. In these experiments Cruse and collaborators identified distinct leg coordination mechanisms and suggest the use of negative and positive feedback to resolve the closed chain kinematics of walking insects. Thus, the controller is highly decentralized and uses the mechanical loop through body and environment to achieve the necessary coupling for smooth motions.

Another interesting property of insects is their ability to tolerate leg loss. Graham [7] showed in experiments with stick

M. Görner and G. Hirzinger are with the Institute of Robotics and Mechatronics at the German Aerospace Center (DLR), Wessling, Germany martin.goerner@dlr.de, gerd.hirzinger@dlr.de
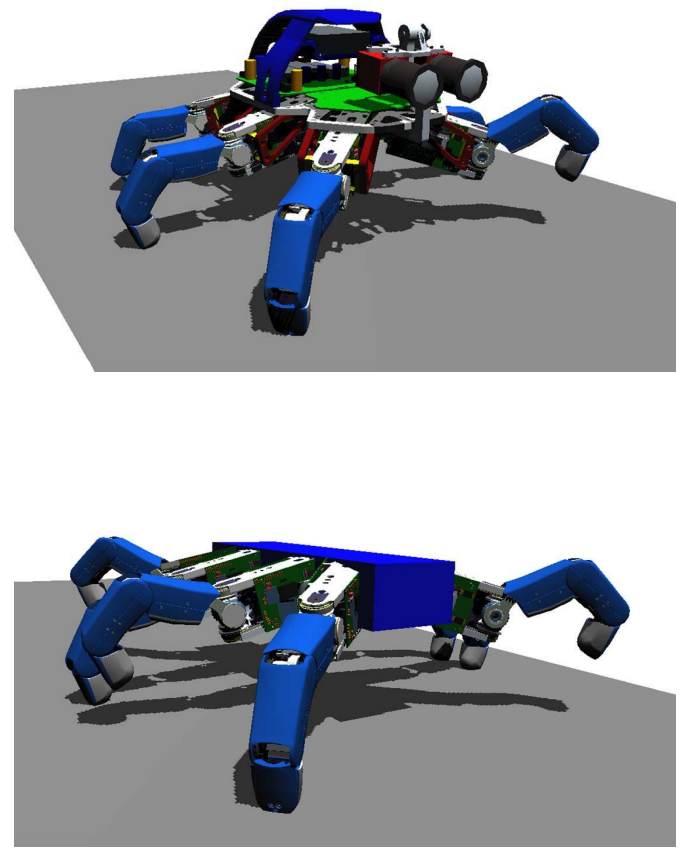

Fig. 1. Top: DLR Crawler (Hexapod) having the left front leg L1 disabled; Bottom: Octapod having the left front leg L1 and the right hind leg R4 disabled, Leg R2 performs a step

insects, during which he amputated various combinations of legs, that the insects in case of leg loss quickly adapt a stable walking pattern at a lower walking velocity. Incorporating such a fault-tolerant gait coordination in walking robots is a very desirable feature to fully exploit their redundancy in case of leg damage or leg loss. The Walknet controller already compensates the loss of a front or a hind leg, but not the loss of a middle leg. An extension to the Walknet by Schilling [9] adds a hypothetical coordination mechanism between the hind and the front leg, which is suppressed while the middle leg works properly but allows stable coordination in case of its loss. Experiments with the robot Hannibal at MIT [4] also considered leg loss. One controller, implemented on Hannibal, changes to a slow wave gait once a leg is disabled and only lifts one leg at a time guaranteeing a stable gait coordination. A second controller, inspired by Cruse's mechanisms, uses the disabled leg as a switchboard that transmits wait or go messages 
from one leg to the next adjacent leg, which also results in stable coordination. The hexapod Oscar [3] employs a similar method as Hannibal to handle leg loss. For eight-legged robots different combinations of the loss of two legs were tested on the Scorpion robot [12]. Herein, various predefined hexapod gait patterns are used to coordinate the walking and were tested for stability and performance. The results show that several combinations of disabled legs allow the robot to keep up walking with some deviation of the desired path, while others lead to instability of the robot.

This article presents the extension of the biologically inspired omnidirectional gait [6] of the DLR Crawler [5] to handle leg loss. Using simulations, the effects of leg loss are studied for the actively compliant hexapod with respect to gait coordination stability and motion stability. In order to enhance the motion performance after leg loss, two methods are proposed and discussed. All simulations use the dimensions of the real robot, which has a mass of $3.5 \mathrm{~kg}$, a leg length of $155 \mathrm{~mm}$ and which feet span an area of $350 \times 380 \mathrm{~mm}$ in a common configuration while its body stands $90 \mathrm{~mm}$ above ground. Additionally, this paper briefly presents and analyzes the novel extension of the Cruse inspired leg loss tolerant gait algorithm from six-legged to eight-legged walking robots. After a review of the basic hexapod algorithm in section II, the extended mechanisms to cover leg loss are presented in section III and the effect of leg losses on the emergence of a stable walking pattern is analyzed. In section IV, the stability of locomotion in the event of leg loss is evaluated and the methods proposed to enhance the stability as well as the walking performance are studied. Finally, section V presents the extension of the concept to eight-legged walking.

\section{CRUSE INSPIRED GAIT OF THE DLR CRAWLER}

For a statically stable gait it is important that all legs in stance (performing their power stroke) move in a coordinated way and that they step (perform their return stroke) in an ordered sequence, such that never two directly neighbored legs step at the same time and that the center of gravity always lies within the support polygon.

In case of the DLR Crawler [6] all stance legs move kinematically correct according to the external commands walking direction, walking velocity and angular velocity about the vertical axis. The gait coordination is based on three of the inter-leg mechanisms that Cruse identified for the stick insect [2]. These three mechanisms achieve a flexible gait by influencing the posterior extreme position (PEP) of each leg, i.e. the transition from power to return stroke, while the anterior extrem position (AEP), i.e. the transition from return to power stroke, remains unchanged. The solid arrows in Figure 2 display how the legs interact with each other by use of mechanisms 1,2 and 3. The dashed arrows are artificial influences, which get activated in case of leg loss and are explained in the following section.

Mechanism 1, which is directed towards the front of the Crawler, inhibits the return stroke of a leg while the posterior

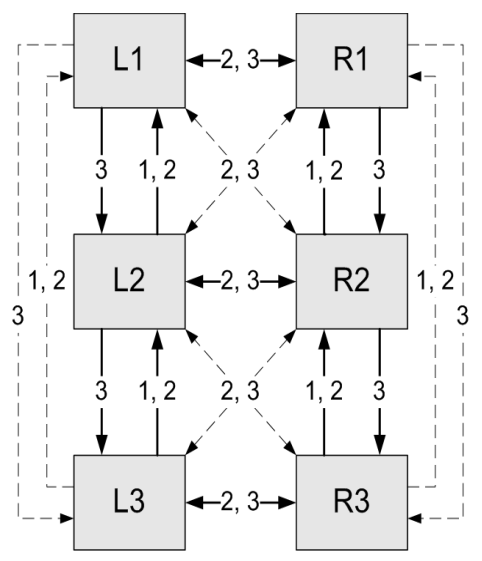

Fig. 2. Cruse' s rules: solid arrows - regular inter-leg influences, dashed arrows - inter-leg influences to cover leg loss

leg performs a return stroke. Mechanism 2, also directed towards the front of the Crawler and additionally coupling contralateral legs, excites the start of the return stroke of a leg for a certain time after the posterior leg changed from return to power stroke. Mechanism 3, directed towards the back of the Crawler and also coupling contralateral legs, excites the start of the return stroke of a leg with increasing strength the closer the anterior leg approaches its PEP. The actual PEP of a leg is calculated as the sum of a nominal PEP and the actual values of all mechanisms influencing the leg multiplied by manually tuned weights. Thus, during walking the PEP of each leg continuously changes.

Commonly the PEP is considered as a distinct distance on a line parallel to the longitudinal axis of an insect or a robot. For the DLR Crawler to allow omnidirectional motion, the PEP and the AEP are implemented as radii of circles rather than distinct points. Whenever a leg crosses its PEP circle, which is shrinking or extending according to the leg coordination mechanisms, it will request to step. Here, an additional artificial mechanism introduced for the DLR Crawler comes into the game. It checks if the direct neighbors are in stance before granting the step. If not, the stance motion of the leg desiring to step is prolonged up to a kinematically useful limit. If after reaching this limit the leg is still not allowed to step, all stance legs are stopped until the situation is resolved. Normally, Cruse's rules prevent that adjacent legs step at the same time, but for some walking commands or in case of a delay due to a reflex situations can occur, during which the return strokes of neighbored legs overlap with respect to time. The additional mechanism guarantees that never two neighbored legs step at the same time and subsequently destabilize the robot. Thus, Cruse's rules are the basis for the emergence of a stable and robust walking pattern, but the artificial mechanism enforces it at all times. Disabling mechanisms 1, 2 and 3, the additional mechanism forces the robot into an omnidirectional tripod gait with overlapping stance phases.

Important to the gait coordination is that all legs perform their return strokes at an equal, high return stroke velocity. This velocity is the speed at which the projection of a foot 
moves along a straight line from its PEP to its AEP during a step with respect to the body coordinate system. It defines the absolute limit for the walking velocity of the robot, which can not walk faster than in a perfect tripod gait at the return stroke velocity. During their return stroke, the legs always target a point on their AEP circle that is calculated at the onset of the return stroke corresponding to the walking commands of the robot.

In the following sections we term a gait coordination a stable coordination, if for a continuous motion a regular pattern emerges, where no directly neighbored legs step at the same time and the robot does not have to stop because one leg has reached its kinematical limit before its neighbor has finished its step. This means for a hexapod that always at least a tripod, i.e. front and hind leg on one side and middle leg on the opposite side, is in stance. A coordination is termed marginally stable coordination, if for a continuous motion a regular pattern emerges after the robot has stopped for a single time because one leg had to wait for another with its return stroke. If multiple stops occur the coordination is termed unstable coordination. An unstable coordination does not necessarily mean that the robot can not walk anymore. In many cases the robot moves apparently smooth with brief stops that are not noticeable to the human eye.

Figure 3 shows which combinations of motion commands result into a stable coordination for the DLR Crawler, stepping with a return stroke velocity of $80 \mathrm{~mm} / \mathrm{s}$. Herein, each marker represents a 60 second kinematic simulation of the Crawler for a distinct velocity command.

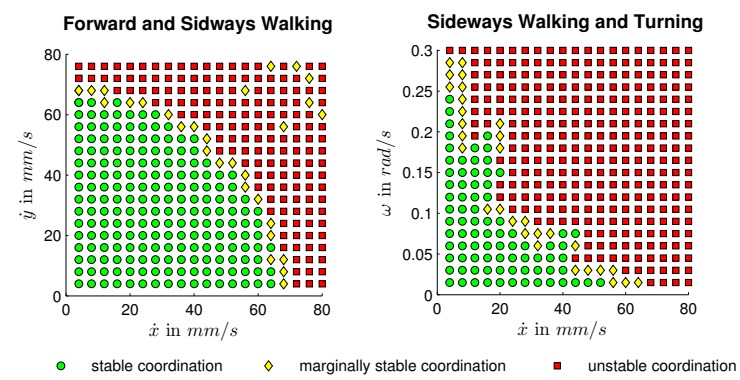

Fig. 3. Gait coordination stability of the regular hexapod $(80 \mathrm{~mm} / \mathrm{s}$ return stroke velocity); Left: forward / sideways walking, Right: forward walking / turning

On the left side of figure 3 the combination of forward walking and sideways walking towards the left is shown. Combinations of forward walking and walking towards the right are omitted since they behave similar and only present a mirrored image. It can be seen that walking commands up to about $60 \mathrm{~mm} / \mathrm{s}$ forward or sideways show a stable gait coordination. The stable region possesses a circular boundary, which means that a stable gait pattern emerges for the robot walking in each direction with commanded velocities up to about $60 \mathrm{~mm} / \mathrm{s}$. At higher velocity commands multiple situations occur that cause the robot to stop walking because one leg has not finished its return stroke, while another leg has reached its preset kinematic limit. The final gait at $60 \mathrm{~mm} / \mathrm{s}$ is a perfectly synchronized tripod with brief double stance phases. The plot also shows some outliers of marginal stability within the unstable region. These are not of further interest since they represent very sensitive states that would turn unstable for small changes of velocity. On the right side of Figure 3 combinations of forward velocity commands and angular velocity commands are shown that result in curve walking. Again only turning to the left is displayed since turning to the right behaves similar. The plot is more scattered and does not show such a nice circular region of stable coordination as the plot on the left. It can be seen that for a broad range of higher forward velocities only small angular velocities are possible, leading to curve walking with larger radii. The boundary region between the stable and unstable region slightly depends on the initial position of the robot. In this case the robot starts from a symmetric initial position where the middle legs are perpendicular to its longitudinal axis and the hind and front legs form an angle of 40 degree with the line connecting the middle legs. If the robot is commanded to stop by a higher control level it returns to this initial position. Thus, plots considering a number of random initial configurations are omitted in this place. Nevertheless, considering different initial conditions would lead to a broader boundary region. Increasing the return stroke velocity of the robot results in larger stable regions of equal shape and was successfully tested for walking velocities up to $200 \mathrm{~mm} / \mathrm{s}$.

\section{GAIT COORDINATION TOLERATING LEG LOSS}

For the following studies concerning leg loss, a lost leg is considered to be "out of the way". This means it is in a position where it does not disturb the motion of the robot, which is valid for robots designed with backdrivable joints or releasable legs. In comparison, it is also possible that a leg gets damaged and remains in an outstretched locked configuration, massively disturbing the locomotion. These cases are not considered within this article.

In order to cope with the loss of a single leg, some additional inter-leg connections have been implemented, shown by the dashed arrows in Fig. 2. These are completely artificial and implement mechanisms 1,2 and 3 in between not adjacent legs. These mechanisms are suppressed as long as all legs work properly and get activated if a leg is labeled as lost or damaged and thus removed from the network. The idea is to continue stable walking by immediately establishing new neighborhood relationships in case of a leg loss and to exploit the full capability of all coordinating mechanisms. This method is somehow related to the work of Schilling, who added mechanism 1 between front and hind leg for the Walknet [9], and the work of Ferrell, who used a damaged leg as a switchboard to transmit messages to its neighbored legs [4], but in contrast uses all three mechanisms and immediately assigns new contralateral and ipsilateral neighborhood relations to retain a stable gait coordination.

Figure 4 exemplarily displays the inter-leg influences in case of the loss of the left front leg or the left middle leg. In case of the loss of a front leg, the middle leg on the same 

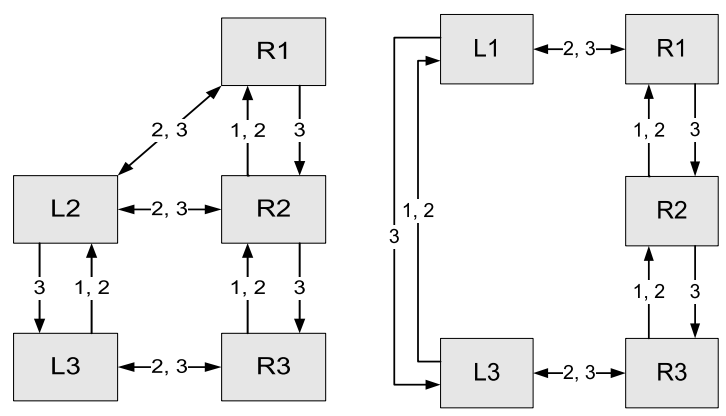

Fig. 4. Left: L1 disabled/lost; Right: L2 disabled/lost

side is treated as the new front leg and is connected to the front leg of the other side by mechanisms 2 and 3. In case of the loss of a middle leg, the front and hind leg of the same side are treated as new neighbors and are connected by all three mechanisms. The loss of a hind leg is treated similar to the loss of a front leg. Figure 5 shows how the hexapod gait immediately adapts to the loss of a leg and keeps up a stable gait coordination.

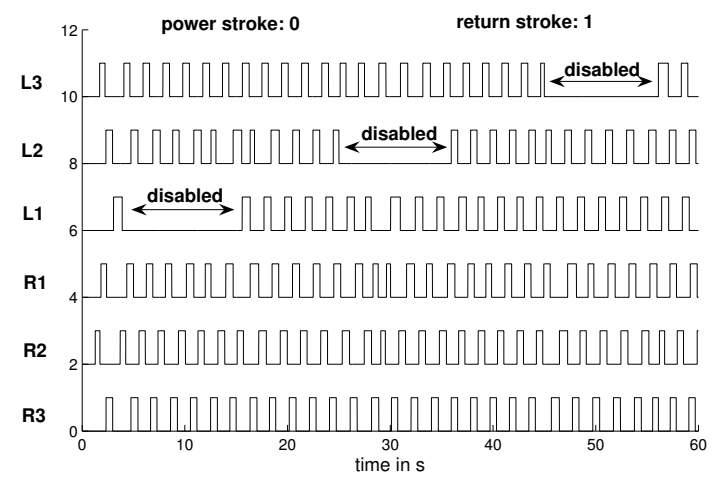

Fig. 5. Gait coordination of the hexapod experiencing the loss of L1, L2 and L3 after each other $(40 \mathrm{~mm} / \mathrm{s}$ forward walking command, $80 \mathrm{~mm} / \mathrm{s}$ return stroke velocity)

It is of interest now to see what influence the loss of a single leg has on the gait coordination and the possible walking velocities given a fixed return stroke velocity. Figure 6 shows the effect of a leg loss for the combinations of forward and sideways velocity commands. Each plot shows the loss of a different leg, where L1 is the left front leg, L2, the left middle leg and L3, the left hind leg. The right side follows the same naming conventions. For the kinematic simulation of the robot the velocity commands were ramped up to the desired combination, following a leg was disabled and the simulation ran for further 60 seconds. For all green markers the robot had at all times a stable gait coordination and the loss of the leg did not disrupt the gait. The gait immediately adjusted to the new situation. The yellow markers again stand for marginal stability of the gait coordination. In most of these cases of marginal stability the

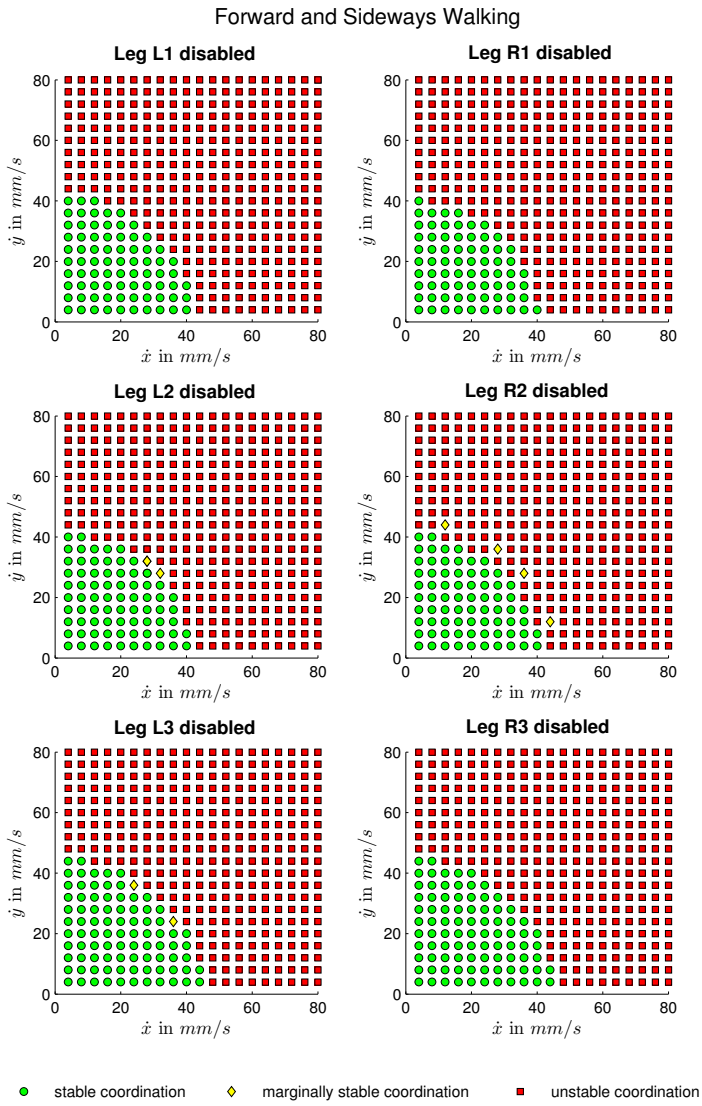

Fig. 6. Gait coordination stability of the hexapod experiencing the loss of a single leg (forward / sideways walking, $80 \mathrm{~mm} / \mathrm{s}$ return stroke velocity)

robot had to stop briefly after the leg loss because one leg had to wait for its neighbor to finish its return stroke. Afterwards it resumed stably coordinated five-legged walking. For the red markers multiple stops had been evoked during the 60 seconds simulation run. As already mentioned above for the cases of unstable coordination the robot also moves forward, but more or less smooth. Further, the gait phase at which the leg loss occurs plays some role for the coordination stability considerations. There are configurations where one leg is close to the switch to its return stroke when its direct neighbor is damaged or lost. Due to this event the leg has to delay its return stroke and to prolong its stance phase such that it reaches its kinematic limit. As a consequence the whole robot has to stop. After this instant a stable coordination is regained. This behavior is especially apparent for the velocity combinations at the boundary of the stable region and means that yellows markers could be green and green markers could be yellow if the switch would have occurred at a different time. Nevertheless, it can be seen from the diagrams that the algorithm shows a similar circular region of stable gait coordination for all different cases of single leg loss. For all plots the new walking velocity limit is roughly at $40 \mathrm{~mm} / \mathrm{s}$ in each direction having a return stroke velocity of $80 \mathrm{~mm} / \mathrm{s}$.

A similar behavior can be observed in figure 7 for the loss 

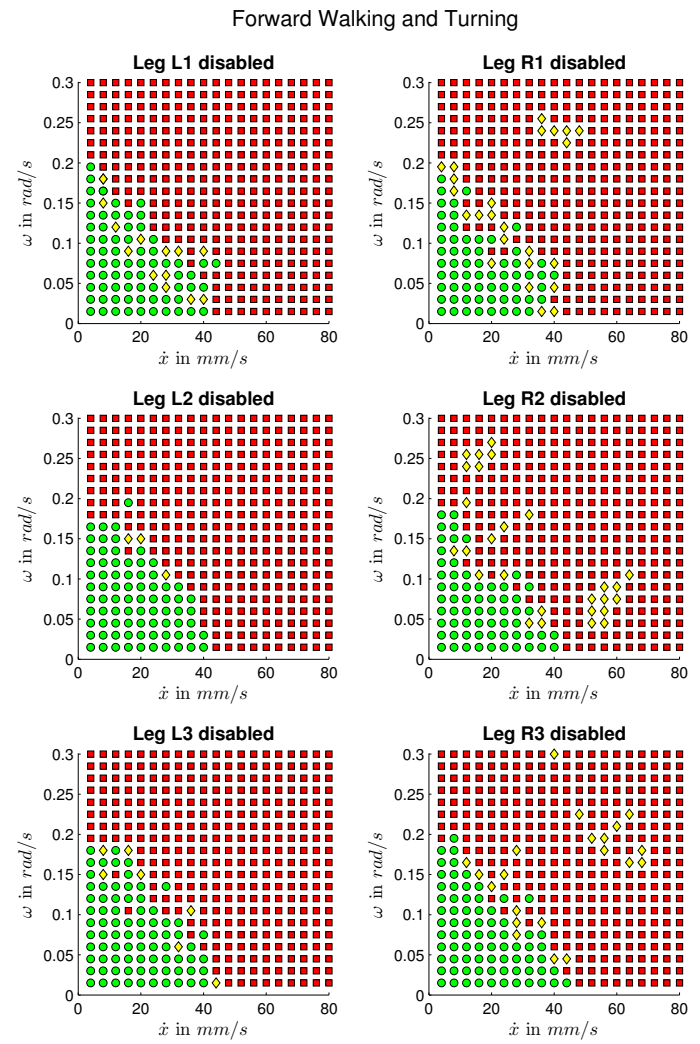

- stable coordination $\diamond$ marginally stable coordination

- unstable coordination

Fig. 7. Gait coordination stability of the hexapod experiencing the loss of a single leg (forward walking / turning, $80 \mathrm{~mm} / \mathrm{s}$ return stroke velocity)

of a leg during curve walking. Here approximately triangular regions of stable coordination appear for the combination of angular and forward velocity commands. In comparison to forward and sideways walking the boundary region is broader and thus stronger restricts the possible motion commands. The circular and triangular shapes of the stable regions are a nice feature, which allows to formulate simple rules for the reduction of the external velocity command in case of a leg loss. Further, it is appealing that the gait coordination behavior shows little dependence on which leg is lost. The gait coordination adaptation also accounts for the loss of two legs. Nevertheless, this is only useful for the loss of both middle legs, since all other combinations lead to longer periods of static instability.

\section{STABILITY OF WALKING SUFFERING LEG LOSS}

Despite the kinematic considerations of a stable coordination, which aims at a smooth and continuous gait always having at least a tripod forming the polygon of support, stability in a dynamic sense plays an important role for walking robots. Regaining a stable gait coordination after leg loss does not necessarily prevent strong tilting motions including body impacts with the ground. Nevertheless, finding a formal definition for the dynamic stability of walking for multi-legged robots is a difficult problem and no promising approaches are know to the authors. It is not the goal of this work to find a formal definition but to refer to some behaviors which are undesirable during walking.

For a walking robot it is desirable that it follows a commanded path with little deviation. Also, considering visual navigation a shaky motion experiencing strong pitching or rolling should be avoided. Ground impacts of the robot body are undesired since they might cause damage. Further, strong limping, and thus large stress on the legs, should be avoided. In order to evaluate the robots walking capability when a leg is lost the following heuristic measures are considered,

- the curvature and length of a path traveled during a certain time span,

- the periodically appearing minimum and maximum pitch angles of the body,

- the periodically appearing minimum and maximum roll angles of the body.

The second and third measure do not consider the peak pitch and roll angles right after the leg loss when the robot occasionally stumbles but consider the regular tilting after the gait has adapted to the leg loss.

\section{A. Simulation Model}

To test for the measures a simplified dynamic simulation model is used. The simulated robot has the dimensions of the DLR Crawler and walks in a plane without obstacles. Each leg has three active joints and one distal passive joint, which is coupled one to one to the last active joint. The robot body has an uniformly distributed mass of $3.5 \mathrm{~kg}$, while the legs are modeled massless. This assumption is valid, since the motions are relatively slow and the mass of the moving parts of a leg is small compared to the body mass. The joints include a controllable passive compliance in form of a serial spring-damper-system connecting motor and joint. This results in a similar behavior to the active joint compliance controllers of the real DLR Crawler. For the simulation the motor side of the spring-damper-system of each joint is perfectly following the desired joint trajectory. During the swing phase, the leg is assumed to follow its desired trajectory. Once in contact with the ground, the contact point is stored and the leg joint springs are loaded. This means the real foot position at the contact point deviates from the desired foot position, virtually penetrating the ground. For both, the desired foot position and the actual position at the contact point, the corresponding joint angles are calculated by inverse kinematics. The difference of desired to actual joint angle represents the loading of the joint spring and allows to calculate the joint torque. Knowing the joint torques the ground contact forces can be calculated using the leg Jacobian and are applied to the robot body. This moves the body and the attachment point of the legs at the body and changes the leg configurations. The contact is further monitored for slipping. Once, the tangential contact force is larger than the friction force, a first order sliding model is applied to the contact point. If the contact breaks the leg follows again its desired trajectory. In order to verify the 
used simplified dynamic model with massless legs, simulations were run using the commercial multi-body simulation software SIMPACK and a model with an equal total mass and leg masses of $0.1 \mathrm{~kg}$. The results of both simulations showed only marginal deviations, thus verifying the assumptions of the simplified model.

\section{B. Analysis of the Motion Stability}

The simulations were run for two different forward walking velocity commands, $20 \mathrm{~mm} / \mathrm{s}$ and $40 \mathrm{~mm} / \mathrm{s}$, which lead to stable coordination according to the above results. Three different leg loss situations have been considered, the left front leg, L1, the left middle leg, L2, and the left hind leg, L3. For the case of leg loss, the leg was lifted and remained in this "out of the way" position not disturbing the robots motion. The simulation was run for each velocity command for a time span that theoretically allowed the robot to travel 2 $\mathrm{m}$ forward with respect to the fixed world coordinate system.
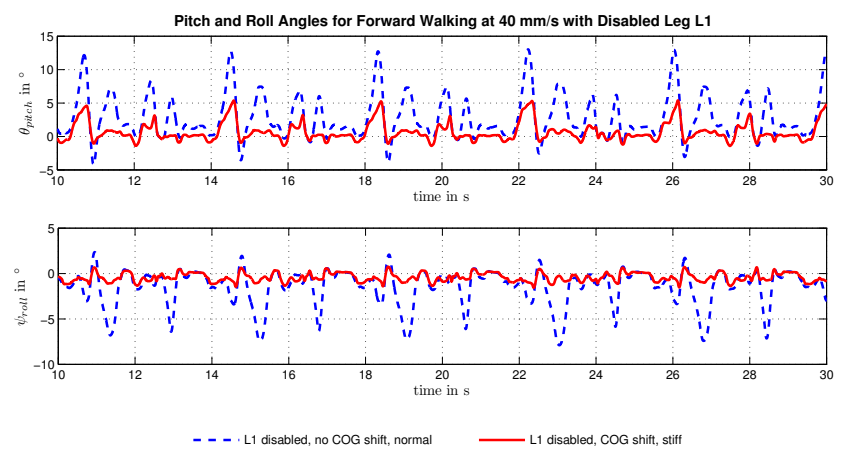

Fig. 8. Pitch and roll angles for the loss of leg L1;

As shown by the blue dashed graphs in Figure 8 , the loss of a leg induces strong tilting motions that degrade the stability and the walking performance of the robot. If like in this example the left front leg L1 is lost, the robot pitches downwards and rolls to the left during the step of the left middle leg L2. This happens due to the fact that the projection of the COG temporarily leaves the support polygon. Thus, having a stable gait coordination does not imply static stability. In order to reduce the tilting and to improve the walking performance in case of leg loss, several changes to the robot configuration have been implemented and evaluated. These changes aim to improve the force distribution without explicit calculation. The first is a shift of the robots center of gravity away from the lost leg by adding an offset to all desired foot positions. In case of a loss of the left front leg the robot body moves $20 \mathrm{~mm}$ backwards and $20 \mathrm{~mm}$ to the right with respect to its feet. This leads to a better force distribution, which shifts the load away from the lost leg and thus reduces the tilting motions. The red solid graphs in Figure 8 exemplarily show the reduction in pitching and rolling for a shifted COG and stiffened joints, where most of the improvement results of the COG shift. Implementing a mechanism that shifts the robot COG towards the instantaneous center of the support polygon did not lead to better results than shifting the COG by a fixed value.

A second idea to enhance the walking is to use the variable joint compliance and to stiffen the joint springs of some legs. In this case, the stiffer legs better follow the desired trajectories, while the other legs are softer and allow stronger deviations from their desired paths. Thus, the robot should move into a position that results in a better force distribution. Changing the joint stiffness for different combinations of legs strongly improved the walking performance in some cases while in other cases the performance deteriorated. No consistent rules could be extracted so far, how to change the stiffness of distinct joints to always improve the walking performance. For this reason, the following four cases are compared to each other: 1. - the COG is not shifted and the robot is compliant, 2. - the COG is not shifted and the robot is stiff (joint stiffness doubled), 3. - the COG is shifted and the robot is compliant and 4. - the COG is shifted and the robot is stiff.

Figures 9 and 10 display the range of periodically occurring pitch and roll angles of the robot body respectively. It can be seen that especially the pitching motion depends on the walking velocity. At lower velocities the loss of L3 causes the strongest effects, while at higher velocities the loss of L1 is more severe. Shifting the COG improves the behavior for all cases but has only little effect for the loss of L2. Additionally stiffening of the robot joints leads to further reductions of the pitching and rolling motions, but is not efficient if the COG is not shifted. Thus, shifting the $\mathrm{COG}$ of the robot in case of leg loss mainly improves the stability in terms of reducing the pitch and roll angles.

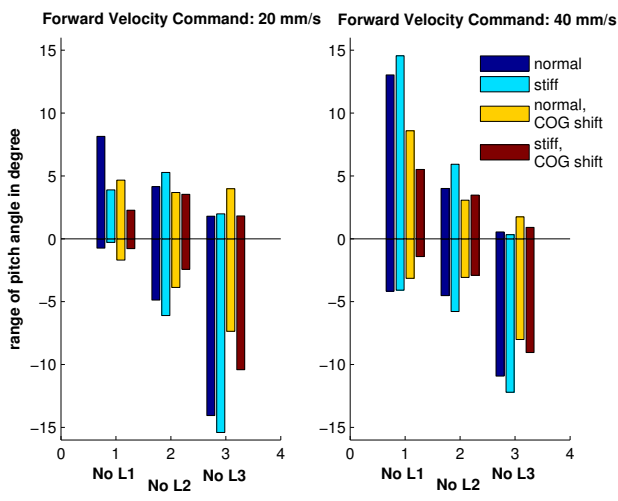

Fig. 9. Comparison of the pitch angles for the loss of L1, L2 or L3; Left: at a commanded velocity of $20 \mathrm{~mm} / \mathrm{s}$ (simulation time: $120 \mathrm{~s}$ ), Right: at a commanded velocity of $40 \mathrm{~mm} / \mathrm{s}$ (simulation time: $60 \mathrm{~s}$ )

Figure 11 gives a good example for the effect of shifting the COG and stiffening the robot on its walking performance. It can be seen that for slower and faster motions the curvature of the path of the robot is strongly reduced and that the distance traveled is increased.

Generally, shifting the COG is beneficial for the stability and the walking performance, while solely stiffening the robot in parts or complete gives unclear results. Remaining deviations of the desired path in case of leg loss can be 

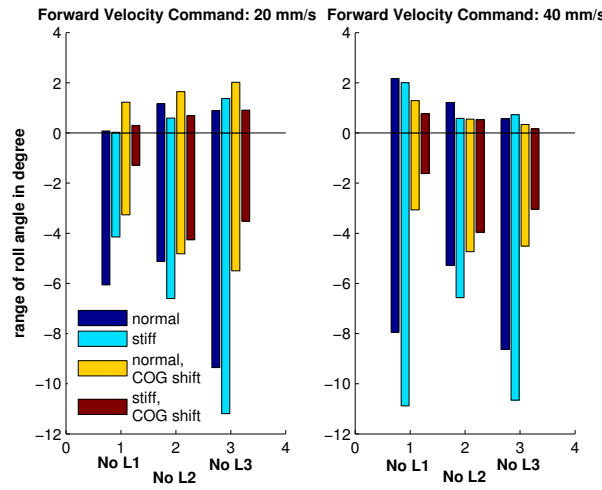

Fig. 10. Comparison of the roll angles for the loss of L1, L2 or L3; Left: at a commanded velocity of $20 \mathrm{~mm} / \mathrm{s}$ (simulation time: $120 \mathrm{~s}$ ), Right: at a commanded velocity of $40 \mathrm{~mm} / \mathrm{s}$ (simulation time: $60 \mathrm{~s}$ )

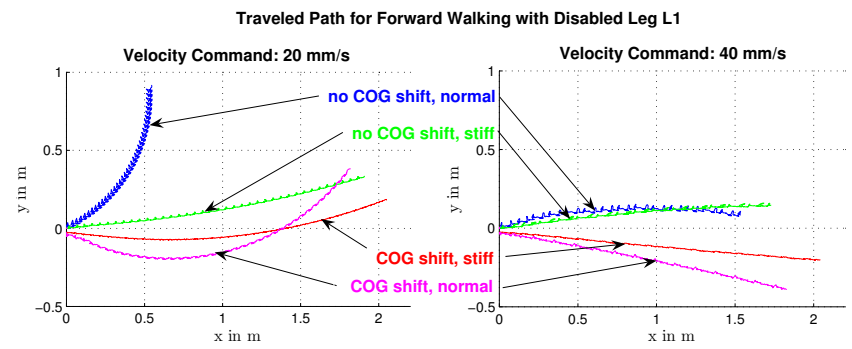

Fig. 11. Walking performance for the loss of leg L1; Left: at a commanded velocity of $20 \mathrm{~mm} / \mathrm{s}$ (simulation time: $120 \mathrm{~s}$ ), Right: at a commanded velocity of $40 \mathrm{~mm} / \mathrm{s}$ (simulation time: $60 \mathrm{~s}$ )

further corrected by commanding a small angular velocity offset opposing the curvature of the path.

\section{EIGHT-LEGGED}

The above presented biologically inspired leg loss tolerant gait controller for a hexapod can be easily extended to be applied to an eight-legged robot. Just two more legs are connected to the network using the three mechanisms as shown by the solid arrows in Figure 12. The dashed arrows again show the artificial mechanisms to cover the loss of multiple legs as exemplarily shown in Figure 13 for the loss of $\mathrm{L} 1$ and R3.

For the eight-legged robot, walking with a return stroke velocity of $80 \mathrm{~mm} / \mathrm{s}$, a stable gait emerges for similar velocity commands as for the hexapod. In case of the loss of a single leg the plots showing the gait coordination stability for forward and sideways walking show the same circular stable regions as for the six-legged robot. Just for the loss of a middle leg, the regions are slightly larger. Considering the stable regions for forward walking and turning, the plots are qualitatively equal, but the stable regions appear at lower angular velocities. This is a result of the larger dimensions of the eight-legged robot, while having equally sized legs. This means that turning at the same speed, the Cartesian velocities of the outer legs are higher for the eight-legged than for the six-legged, thus reaching their limits at lower angular velocities. Because of the limited space and similar

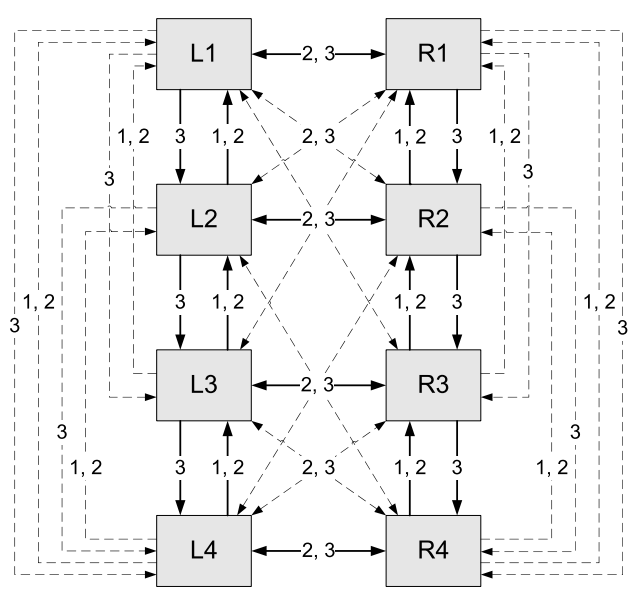

Fig. 12. Cruse' s rules for the eight-legged robot: solid arrows - regular inter-leg influences, dashed arrows - inter-leg influences to cover leg loss

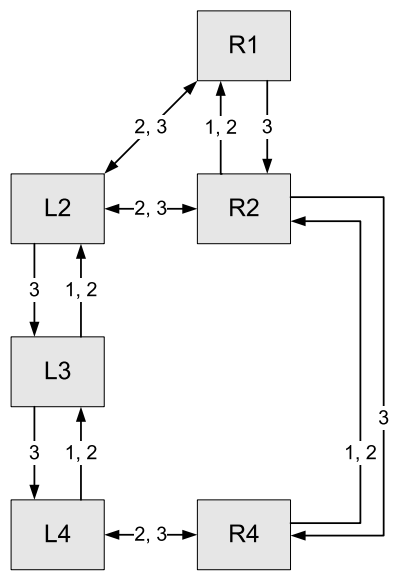

Fig. 13. Legs L1 and R3 disabled/lost

results compared to the six-legged robot the plots are omitted in this article. Figure 14 shows that the octapod immediately adapts to the loss of each single leg and keeps up a stable gait coordination. The loss of a single leg has only very little effect on the stability of walking and the simulated robot shows only very little deviation from a desired straight line path. Also the pitching and rolling motions are negligible.

In comparison to the hexapod, the octapod can handle the loss of two legs a lot easier. In most cases the loss of two legs impairs the motion less than the loss of a single leg impairs the motion of the hexapod. The loss of a pair of middle legs is handled most easily. The simulated robot even adjusts its motion to the loss of both front or both hind legs without experiencing strong tilting or rolling. It only moderately deviates of a desired straight line path. This behavior can be explained by the fact that the vertical projection of the COG almost never leaves the support polygon, thus keeping up static stability. One configuration that is less stable occurs, if a front or hind leg and the directly neighbored leg on the 


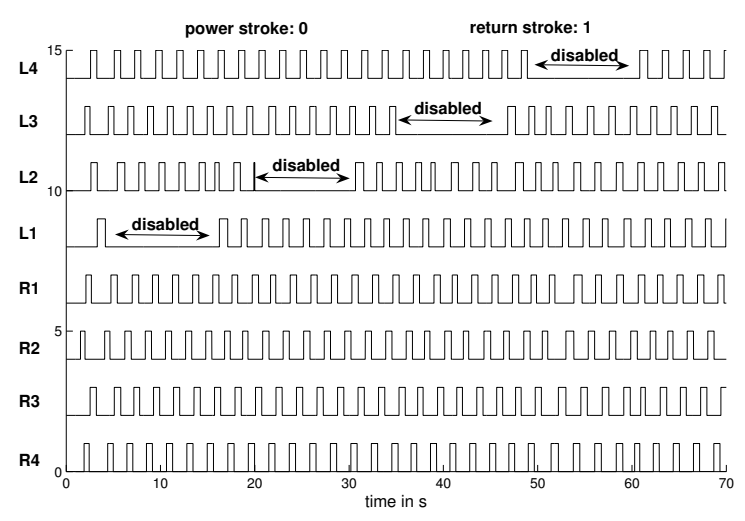

Fig. 14. Gait coordination of the octapod experiencing the loss of L1, L2, L3 and L4 after each other $(35 \mathrm{~mm} / \mathrm{s}$ forward walking command, $80 \mathrm{~mm} / \mathrm{s}$ return stroke velocity)

same side are lost. In these cases a strongly curved path can be observed, which again can be improved by shifting the COG away from the lost legs. Figure 15 shows stable gait patterns emerging for different situations of a loss of two legs. It can be summarized that an eight-legged robot easily handles the loss of a single leg and the loss of two legs without being strongly impaired. Even the loss of three or four legs can be handled in some cases. Due to the multitude of possible leg loss situations for an eight-legged robot and limited space, a detailed analysis can not be presented here.

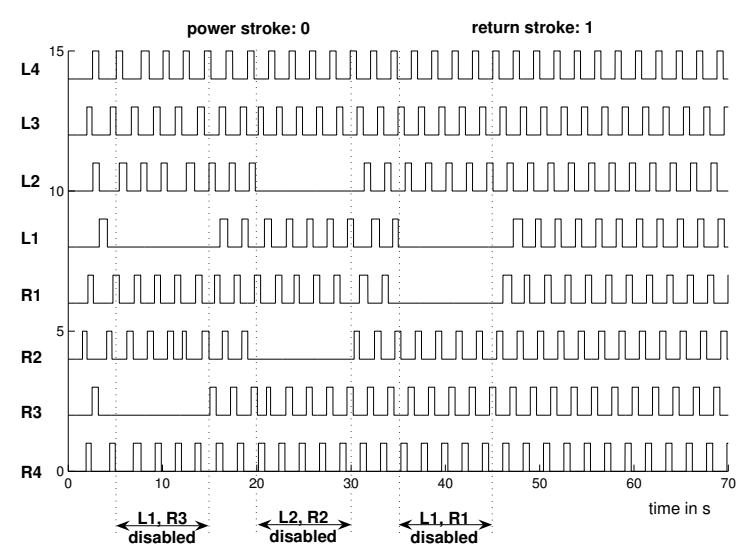

Fig. 15. Gait coordination of the octapod experiencing the loss of two legs at a time $(35 \mathrm{~mm} / \mathrm{s}$ forward walking command, $80 \mathrm{~mm} / \mathrm{s}$ return stroke velocity)

\section{CONCLUSIONS AND FUTURE WORKS}

\section{A. Conclusions}

In this article the Cruse inspired omnidirectional gait of the DLR Crawler is extended to tolerate leg loss. It is shown that the gait immediately adapts to the loss of a leg and that a stable gait pattern emerges despite of which leg is lost. In case of the loss of a leg, the possible velocity commands leading to a stable gait coordination reduce about roughly $35 \%$ compared to the undamaged robot. Further, this range of possible commands is nearly independent of which leg is lost. Dynamic simulations show that the compliant robot experiences curve walking for straight line commands as well as pitching and rolling motions in case of a leg loss. These motions are most severe for the loss of a front or a hind leg. In order to enhance the motion stability and performance a shift of the COG and an adjustment of the joint stiffness are discussed. The COG shift generally improves the motion performance and stability, while the joint stiffness adjustment sometimes improves but in some cases even degrades the performance. Finally, the whole concept of a Cruse inspired leg loss tolerant gait is extended to eight-legged walking. It is shown that this also results in stable gait coordination that easily tolerates the loss of up to two legs.

\section{B. Future Works}

The leg loss tolerant gait poses some new questions to be answered in the future. One is, how Cartesian compliance controllers effect the motion stability compared to the joint compliance control? Another question is, how the gait behaves for rough terrain? Also, situations with locked legs that disturb the motion should be analyzed.

\section{ACKNOWLEDGMENTS}

The authors gratefully acknowledge the reviewers' detailed and valuable comments.

\section{REFERENCES}

[1] J. Ayers, Underwater Walking, Arthropod Structure \& Development, 2004, Vol. 33, No. 3, pp 347-360

[2] H. Cruse, What Mechanisms Coordinate Leg Movement in Walking Arthropods, Trends in Neuroscience, 1990, Vol. 13, pp 15-21

[3] A. El Sayed Auf, F. Mösch, M. Litza, How the Six-Legged Walking Machine OSCAR Handles Leg Amputations, Proceedings of the 9th International Conference on Simulation of Adaptive Behaviour, Rom, Italy, 2006

[4] C. Ferrell, A Comparison of Three Insect-Inspired Locomotion Controllers, Robotics and Autonomous Systems, 1995, Vol. 16, No. 2-4, pp 135-159

[5] M.Görner, Th. Wimböck, A. Baumann, M. Fuchs, T. Bahls, M. Grebenstein, Ch. Borst, J. Butterfass and G. Hirzinger, The DLR-Crawler, A Testbed for Actively Compliant Walking Based on the Fingers of DLR-Hand II, Proceedings of the 2008 IEEE/RSJ International Conference on Intelligent Robots and Systems, Nice, France, 2008, pp $1525-1531$

[6] M. Görner, Th. Wimböck and G. Hirzinger, The DLR Crawler: evaluation of gaits and control of an actively compliant six-legged walking robot, Industrial Robot: An International Journal, 2009, Vol. 36, No. 4, pp 344-351

[7] D. Graham, The Effect of Amputation and Leg Restraint on the Free Walking Coordination of the stick insect Carausius morosus, Journal of Comparative Physiology A, 116, 1977, pp 91-116

[8] T. Kindermann, Behavior and Adaptability of a Six-Legged Walking System with Highly Distributed Control, Adaptive Behavior, 2002, Vol. 9, No. 1, pp 16-41

[9] M. Schilling, H.Cruse and P. Arena, Hexapod Walking: an expansion to Walknet dealing with leg amputations and force oscillations, Biological Cybernetics, 2007, Vol. 96, pp 323-340

[10] M. Schilling, A. Schneider, H. Cruse and J. Schmitz, Local control mechansims in six-legged walking, Proceedings of the 2008 IEEE/RSJ International Conference on Intelligent Robots and Systems, Nice, France, 2008, pp 2655-2660

[11] D. Spenneberg and F. Kirchner, SCORPION: A biomimetic Walking Robot, Robotik 2002, VDI-Bericht, 2002, Vol. 1679, pp 677-682

[12] D. Spenneberg, K. McCullough and F. Kirchner, Stability of Walking in a Multilegged Robot Suffering Leg Loss, Proceedings of the 2004 IEEE International Conference on Robotics and Automation, New Orleans, USA, 2004, pp 2159-2164 